\title{
Prolonged exposure of marine algal phlorotannins with whitening effect did not cause inflammatory hyperpigmentation in zebrafish larva
}

\author{
Seon-Heui Cha ${ }^{1}$, Eun-Ah Kim ${ }^{2}$, Kil-Nam Kim ${ }^{3}$, Soo-Jin Heo ${ }^{2}$, Hee-Sook Jun ${ }^{4-6 *}$ and You-Jin Jeon ${ }^{7 *}$ \\ ${ }^{1}$ Department of Marine Bio and Medical Science, Hanseo Universirty, Republic of Korea \\ ${ }^{2} J$ jeju International Marine Science Center for Research \& Education, Korea Institute of Ocean Science \& Technology (KIOST), Republic of Korea \\ ${ }^{3}$ Chuncheon Center, Korea Basic Science Institute (KBSI), Republic of Korea \\ ${ }^{4}$ College of Pharmacy, Gachon University, Republic of Korea \\ ${ }^{5}$ Lee Gil Ya Cancer and Diabetes Institute, Gachon University, Republic of Korea \\ ${ }^{6}$ Gachon Medical and Convergence Institute, Gachon Gil Medical center, Republic of Korea \\ ${ }^{7}$ School of Marine Biomedical Sciences, Jeju National University, Republic of Korea
}

\begin{abstract}
The demand for novel melanin synthesis inhibitors is increasing due to the weak effectiveness and unwanted side effects. Thus, marine algae have been studied as sources of inhibitors of melanin synthesis, but it is still unclear whether they will be effective in in vivo. In this study, we investigated whether marine algal phlorotannins including phloroglucinol (PG), ekcol (EK), and dieckol (DK) from Ecklonia cava inhibit melanin synthesis in zebrafish embryos. PG, EK, and DK treatment were found to inhibit melanin synthesis in zebrafish embryos as similar to arbutin, used as a positive control. Interestingly, PG, EK, and DK treatment showed no toxicity, whereas arbutin treatment showed toxicity for long-term exposure. As well, mRNA expression of pro-inflammatory cytokines, interleukin-1 $\beta$ (IL-1 $\beta)$, tumor necrosis factor- $\alpha$ (TNF- $\alpha$ ), and cyclooxygenase-2 (COX-2) were increased in arbutin treatment, whereas, the mRNA expression were not altered in PG, EK, and DK treatment by long-tern exposure. These findings suggest that PG, EK, and DK from E.cava inhibit melanin synthesis similar to arbutin without inflammatory toxicity. PG, EK, and DK from E. cava could be a potential prevention and/or therapeutic ingredients for hyperpigmentation.
\end{abstract}

\section{Introduction}

Melanin is the major pigment which is largely responsible for the color of human skin. It has been known to be overproduced in skin hyperpigmentation diseases including post-inflammatory hyperpigmentation after acne [1], and chronic sun exposure.

Several of the known natural melanin synthesis inhibitors, including kojic acid and arbutin, have already been the focus of studies, and are currently being utilized as cosmetic additives [2]. However, it is clearly necessary to find safer and more effective skin-whitening agents, due to the carcinogenic potential and weak whitening effects of kojic acid [3], and the cytotoxicity by UV-irradiation [4] and mutagenicity [5] potential of arbutin. Thus, although, many melanin synthesis inhibitors are currently available, the demand for novel melanin formation inhibitors are increasing.

Marine algae are known to be rich in vitamins, minerals, polysaccharides and polyphenols with valuable pharmaceutical and biomedical potential [6-10].

Phlorotannins isolated from Ecklonia cava are phenolic secondary metabolites such as phloroglucinol, eckol, dieckol, eckstolonol and triphlorethol-A; these phlorotannins have been reported with various biological activities such as angiotensin converting emzyems inhibitory [11], neuroprotection [12], anti-inflammatory [13], and antioxidative $[14,15]$.
Zebrafish has melanin pigments on the surface of their skin, allowing simple observation of the pigmentation process without complicated experimental procedures [16]. Skin melanin of zebrafish could be seen at approximately 24 -hour post-fertilization (hpf). These characteristics suggest that the zebrafish would be an excellent model for the study of melanin synthesis. Therefore, in the present study, we investigated the melanin synthesis inhibitory effects of marine algal phlorotannins including PG, EK, and DK in zebrafish embryos.

\section{Materials and methods}

\section{Zebrafish maintenance}

Adult zebrafish were obtained from a Seoul aquarium (Seoul, Korea) and 10 fishes were kept in $3 l$ acrylic tank. Zebrafish maintained according to previously described [17]. The adult zebrafish procedures

${ }^{*}$ Correspondence to: You-Jin Jeon, School of Marine Biomedical Sciences, Jeju National University, Jeju, Korea, Tel: +82647543475; Fax: +82647563493; E-mail: youjinj@jejunu.ac.kr; youjin2014@gmail.com

Hee-Sook Jun, College of Pharmacy , Gachon Universitym, Incheon Korea, Tel:+82328996056; E-mail:hsjun@gachon.ac.kr

Key words: seaweed, melanin synthesis inhibitor, zebrafish

Received: February 15, 2020; Accepted: February 26, 2020; Published: February 28,2020 
used in the present study were conducted according to the guidelines established by the Jeju National University Ethics Review Committee for Animal Experiments and approved by the Ethical Committee (IEC) of the Jeju National University.

\section{Preparation of phlorotannins from brown alga, Ecklonia cava}

The marine brown alga, E. cava was harvested along the coast of Jeju Island, Korea, between October 2013 and March 2014. The sample was washed three times with tap water to remove the salt, epiphytes, and sand attached to the surface. After then carefully rinsed with fresh water, and maintained in a medical refrigerator at $-20^{\circ} \mathrm{C}$. Thereafter, the frozen samples were lyophilized and homogenized with a grinder prior to extraction. PG, EK, and DK were isolated as previously described $[18,19]$ and its chemical structure was presented in Figure 1A.

\section{Treatment of zebrafish embryos with compounds}

Embryos ( $\mathrm{n}=25)$ were transferred to 12 -well plates and maintained in $1 \mathrm{ml}$ of embryo media $(0.003 \%$ sea salt, $0.0075 \%$ calcium sulfate). Embryos were incubated with or without arbutin, PG, EK, and DK (50 $\mu \mathrm{M})$ from 9-35 hours post-fertilization (hpf) or from 1-5 days post-fertilization (dpf), thereafter the embryos were rinsed in embryo media and anaesthetized using 2-phenoxy ethanol (Sigma, St. Louis, MO) before experiments. Dimethyl sulfoxide (DMSO, Duchefa Biochemie, Netherlands) used for vehicle. Images were observed using the microscope (Carl Zeiss, Germany).

\section{Measurement of melanin contents}

Embryos $(n=25)$ were transferred to 12 -well plates and maintained in $1 \mathrm{ml}$ of embryo media $(0.003 \%$ sea salt, $0.0075 \%$ calcium sulfate). Embryos were incubated with or without arbutin, PG, EK, and DK (50 $\mu \mathrm{M}$ ) from 9-35 hours post-fertilization (hpf), thereafter the embryos were rinsed in embryo media and sonicated in Pro-Prep protein extraction solution (Intron, Korea). Melanin content was determined as described previously [20].

\section{Measurement of heart rates}

The heart rates were measured at $5 \mathrm{dpf}$ of the experiment to determine the toxicity of compounds [20]. Counting and recording of atrial and ventricular contractions were performed for 3 min under a microscope (SZX7, Olympus, Japan), and results were presented as the average heart rate per min.

\section{qRT-PCR}

Total RNA was extracted from zebrafish embryos using RNAiso plus (Takara Bio Inc., Japan), and cDNA was prepared using a PrimeScriptTM cDNA synthesis kit (Takara Bio Inc., Japan) according to the manufacturer's instructions. cDNA samples were analyzed by the SYBR Premix Taq ${ }^{\text {TM }}$, ROX plus (Takara Bio Inc., Japan) on BioRad cyclers (Hercules, CA). Gene expression was normalized to the endogenous housekeeping control gene, $\beta$-actin. Relative expression

Table 1. Primer sequences

\begin{tabular}{|c|c|c|}
\hline Gene name & Sequence & \\
\hline IL-1 $\beta$ & $\begin{array}{l}\text { Forward } \\
\text { Reverse }\end{array}$ & $\begin{array}{l}5^{\prime} \text { - TCAAACCCCAATCCACAGAG- } 3^{\prime} \\
5^{\prime} \text { - TCACTTCACGCTCTTGGATG- } 3^{\prime}\end{array}$ \\
\hline TNF- $\alpha$ & $\begin{array}{l}\text { Forward } \\
\text { Reverse }\end{array}$ & $\begin{array}{l}5^{\prime} \text { - AGAAGGAGAGTTGCCTTTACCGCT- } 3^{\prime} \\
5^{\prime} \text { - AACACCCTCCATACACCCGACTTT- }{ }^{\prime}\end{array}$ \\
\hline $\mathrm{COX}-2$ & $\begin{array}{l}\text { Forward } \\
\text { Reverse }\end{array}$ & $\begin{array}{l}5^{\prime} \text { - AGCCCTACTCATCCTTTGAGG - } 3^{\prime} \\
5^{\prime} \text { - TCAACCTTGTCTACGTGACCATA - } 3^{\prime}\end{array}$ \\
\hline$\beta$-actin & $\begin{array}{l}\text { Forward } \\
\text { Reverse }\end{array}$ & $\begin{array}{l}5^{\prime} \text { - AATCTTGCGGTATCCACGAGACCA - } 3 \\
5^{\prime} \text { - TCTCCTTCTGCATCCTGTCAGCAA - } 3\end{array}$ \\
\hline
\end{tabular}

was calculated for each gene using the $\Delta \Delta$ CT (where CT is the threshold cycle) method. The primer sequences used are listed in Table 1.

\section{Statistical analysis}

All measurements were made in triplicate and all data are represented as mean \pm S.E. The results were subjected to an analysis of variance using the Tukey test to analyze the differences among treatments. Values of $\mathrm{p}<0.05$ were considered significant.

\section{Results}

\section{PG, EK, and DK inhibit melanin synthesis in zebrafish embryo}

First, to determine the melanin formation inhibitory ability of PG, EK, and DK in zebrafish embryos, $50 \mu \mathrm{M}$ of arbutin as a control, which widely used for melanin formation inhibitor, PG, EK, and DK were used. As expected, arbutin showed significant melanin synthesis inhibition. Interestingly, when the zebrafish embryos were treated with PG, EK, and DK showed significant inhibition of melanin synthesis similar to arbutin (Figure 1B), suggesting that PG, EK, and DK are effective inhibitors to melanin synthesis in zebrafish.

\section{PG, EK, and DK have no inflammatory toxicity in zebrafish embryo}

Melanin synthesis inhibitors including arbutin have been reported involved in adverse effects when it used long-term as cosmetics [21]. So, we determined whether PG, EK, and DK show toxicity by longterm exposure in zebrafish embryos. As the results, a significantly lower survival was observed in arbutin-treatment zebrafish embryos (around $50 \%$ survival), interestingly, the treatment of PG, EK, and DK raised the survival similar to the control (Figure 2A). Heart rates is indicator of toxicity in the test. A significantly lower heart rates was observed in arbutin-treated zebrafish embryos, whereas, PG, EK, and DKtreatment did not generate any heat rates disturbance (Figure $2 \mathrm{~B}$ ). In the morphological evaluations, hyperpigmentation was observed with malformation of embryos body including relatively short body length, bow-shaped back, pericardial edema, and bent tail were observed in arbutin-treatment zebrafish embryos by long-term exposure, however, PG, EK, and DK-treatment did not show any adverse effects (Figure 2C). Arbutin is a glucosylated hydroquinone [22], and hydroquinone treatment evoke substantial skin irradiation [23] consequently inflammation induced. So, to determine whether PG, EK, and DK induce inflammation in zebrafish embryos, we examined IL- $1 \beta$, TNF- $\alpha$, and COX-2 mRNA expression after treatment with PG, EK, and DK. mRNA expression of IL- $1 \beta$, TNF- $\alpha$, and COX- 2 were increased (Figure 3 ), as well inflammatory phenotype such as cardiac edema (indicated with arrow in Figure 2C) observed by long-term exposure of arbutin-treatment. On the other hand, the mRNA expression levels were not increased (Figure 3 ), as well there was no observation of phenotypical malformation by longterm exposure of PG, EK, and DK treatment (Figure 2C). These results suggest that PG, EK, and DK treatment have no toxicity and induction of inflammation by long-term exposure in zebrafish embryos.

\section{Discussion}

In the previous studies, a variety of materials has been developed and currently utilized as cosmetic additives or as medicinal products for the treatment of skin hyperpigmentation [24]. However, recent evidence indicate that skin-whitening agent such as hydroquinone; arbutin causing irritation and pain [23]. Therefore, finding safer melanin synthesis inhibitors are extremely important. In the present study, we provide compelling evidence of PG, EK, and DK as a potential 
A<smiles>Oc1cc(O)cc(O)c1</smiles>

Phloroglucinol (PG)<smiles>Oc1ccc(Oc2c(O)c(O)cc(O)c2Oc2cc(O)cc(O)c2)cc1</smiles>

Eckol (EK)

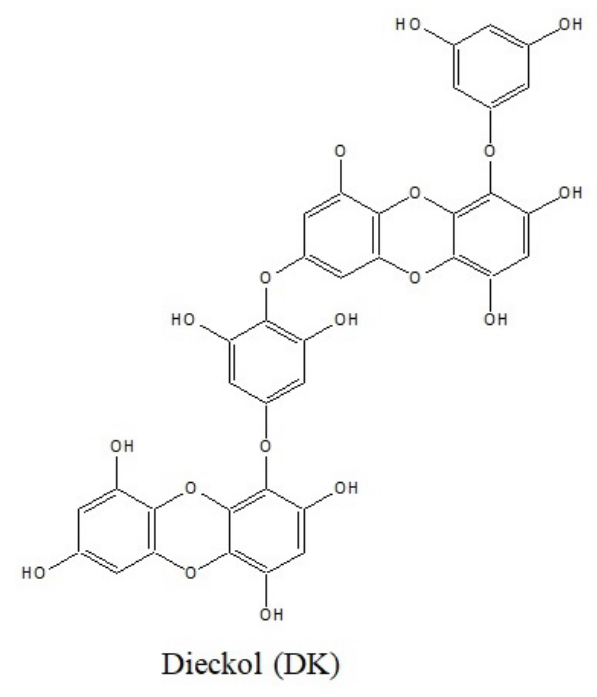

\section{B}
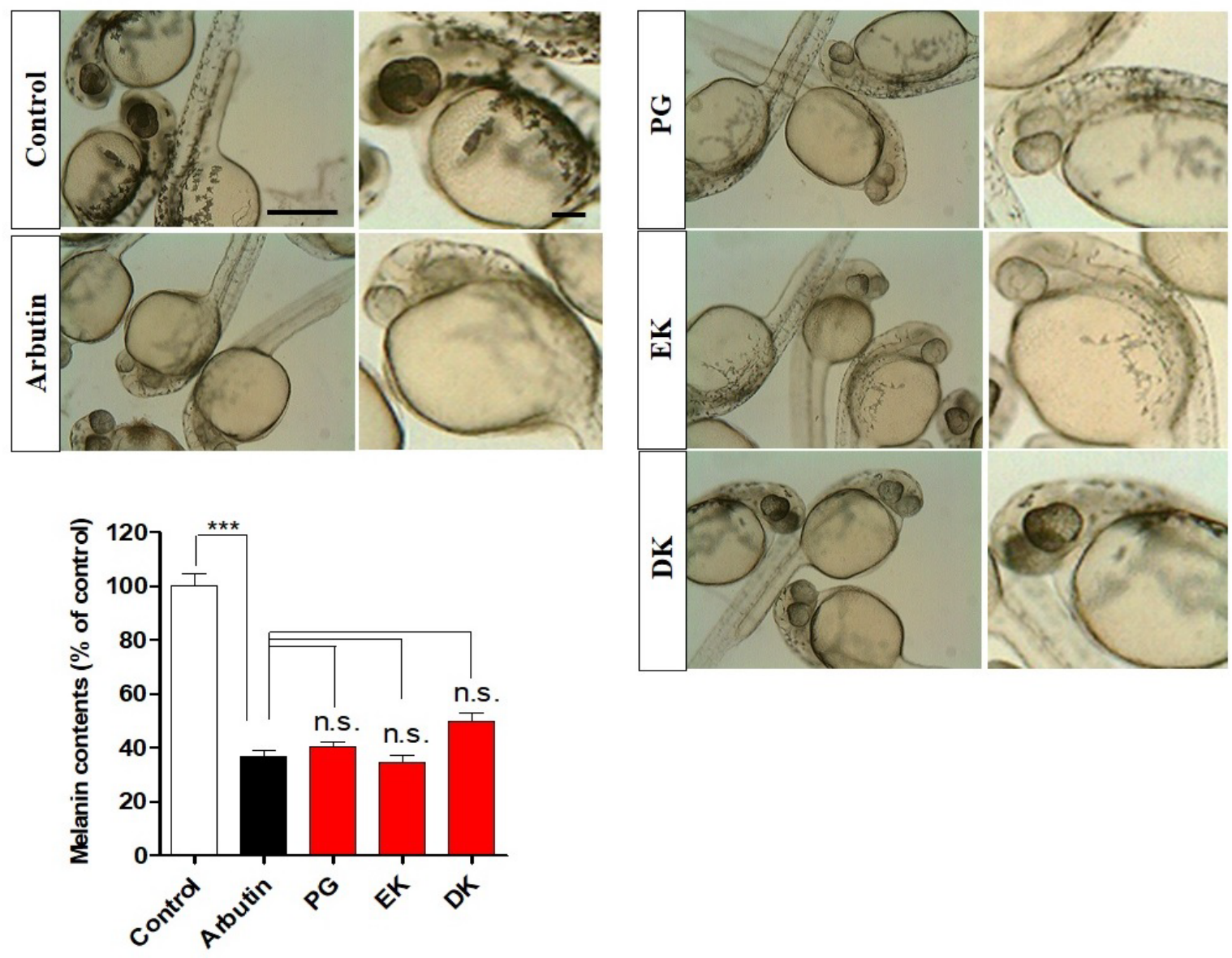

Figure 1. Phlorotannins from Ecklonia cava inhibited melanin synthesis in zebrafish embryos. A. Chemical structure of phlorotannins including phloroglucinol (PG), eckol (EK), and dieckol (DK) isolated from Ecklonia cava. Zebrafish were incubated with arbutin, PG, EK, and DK from 9-35 hours post-fertilization. B. Representative photographs of melanin expression. Scale bar: $500 \mu \mathrm{m}$. Melanin contents were subsequently performed as described in "Materials and Methods". PG: phloroglucinol, EK: eckol, DK: dieckol. Scale bar: $100 \mu \mathrm{m}$. ***p $<0.001$, n.s.: no significance 
A

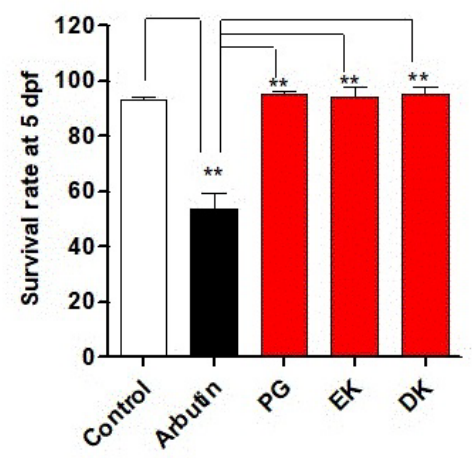

C

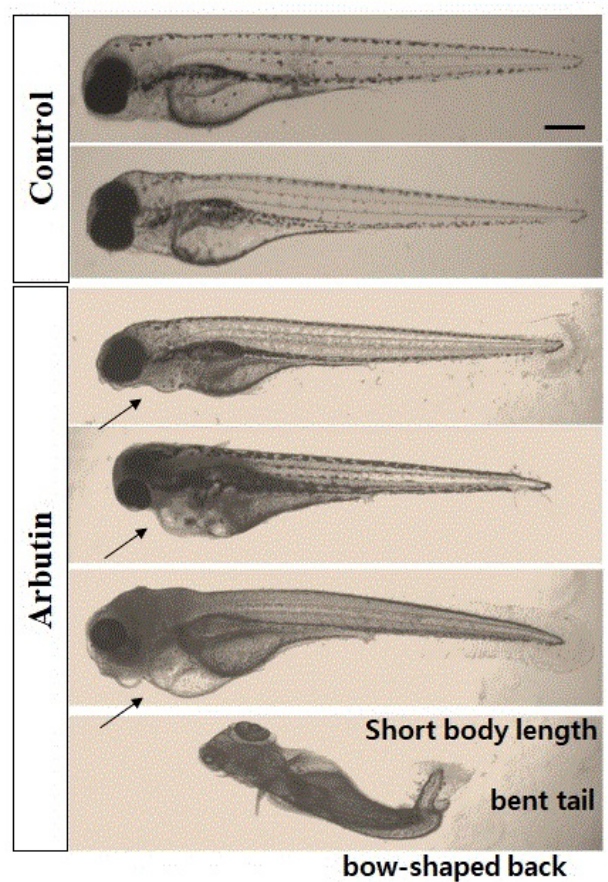

B
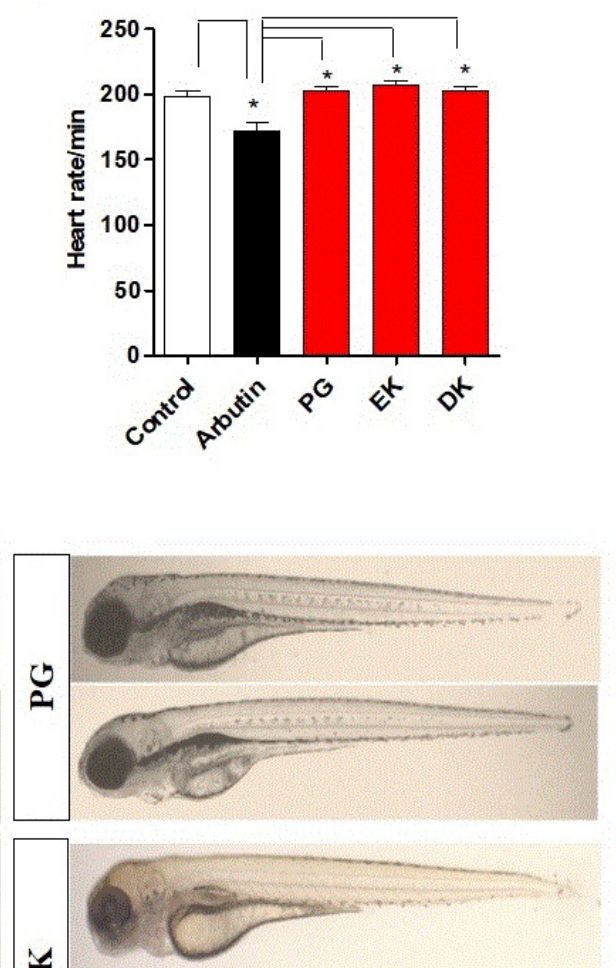

$\stackrel{1}{1}$

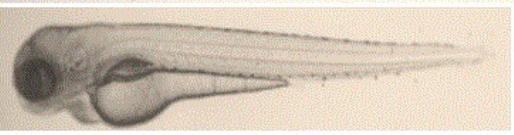

光

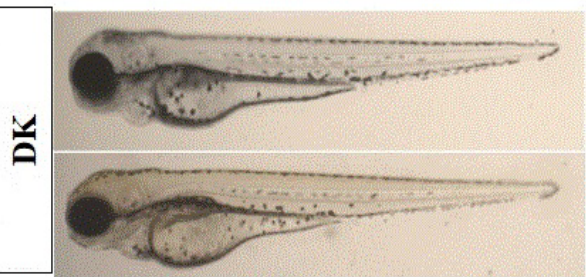

Figure 2. Long-term exposure of phlorotannins from Ecklonia cava have no toxicity in zebrafish embryo. Zebrafish were incubated with arbutin, PG, EK, and DK from 1-5 days postfertilization. A. Survival rate of zebrafish after treated with arbutin, PG, EK, and DK. B. Heart rates of zebrafish after treated with arbutin, PG, EK, and DK. The heart rates were measured at 5 days post-fertilization, the number of heart beats in $3 \mathrm{~min}$ was counted, and the results are expressed as the beats/min. C. Representative photographs of zebrafish embryos after treated with arbutin, PG, EK, and DK. PG: phloroglucinol, EK: eckol, DK: dieckol. Scale bar: $100 \mu \mathrm{m}$. Arrow indicates pericardia. Experiments were performed in triplicate. ${ }^{*} \mathrm{p}<0.05,{ }^{*} \mathrm{p}<<0.01$

A

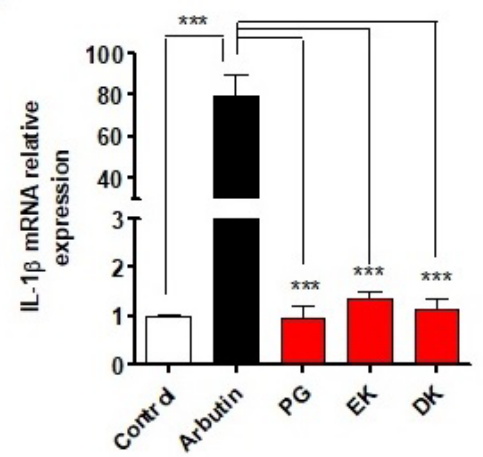

B

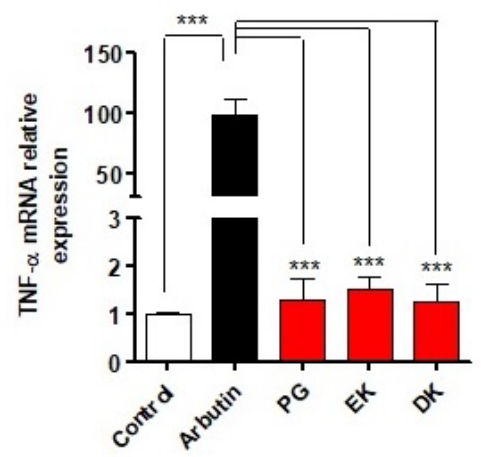

C

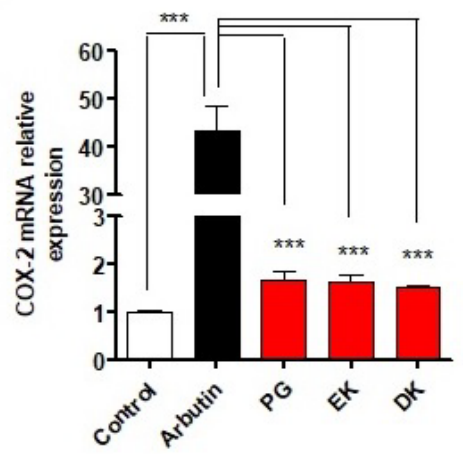

Figure 3. Phlorotannins from Ecklonia cava have no induction of inflammatory cytokine mRNA expression in zebrafish embryos. Zebrafish were incubated with arbutin, PG, EK, and DK from 1-5 days post-fertilization. Total RNA was extracted from zebrafish and mRNA expression levels of: A. IL-1 $\beta$, B. TNF- $\alpha$, and C. COX-2 were analyzed by qRT-PCR. PG: phloroglucinol, EK: eckol, DK: dieckol. $\mathrm{n}=20 \sim 27$ embryos. ${ }^{* * *} \mathrm{p}<0.001$ 
melanin synthesis inhibitor by showing melanin formation inhibition without adverse effects in zebrafish embryos.

Phlorotannins are a class of polyphenolic compounds that have antioxidant capacity and attractive components to study inhibition of melanin formation, are found widely within plants [25,26]. Marine algae have been utilized to maintain good health or to treat various diseases including hyperpigmentation [27-29]. Particularly, marine algae extracts have been reported studies on the melanin synthesis inhibitory effect with inhibition of tyrosinase activity in vivo [20], and, structural compounds isolated from the extracts also have been reported to inhibitory effect of tyrosinase activity and melanin formation in melanocyte [30] and UV protective effect in human fibroblast [31] but it is still unclear whether the effect of the structural materials isolated from the extracts will be effective in in vivo.

In the present result, phlorotannins including PG, EK, and DK isolated from $E$. cava were shown the hypopigmentation potential in zebrafish embryos at a similar level to arbutin, which is a widely used whitening agent. Additionally, the toxicity was found to long-term exposure of arbutin, but not in phlorotannins treatment zebrafish embryo. These results imply that E. cava-derived structural compounds have a whitening effect in in vivo.

A variety of natural or synthetic substances such as hydroquinone such as arbutin are currently utilized as ingredients of preparations designed to control hyperpigmentation, but none of these have proven completely satisfactory, either due to their limited efficacy or owing to safety concerns [23]. Thus, it has recently been banned for cosmetic uses in Europe and some Asian countries [32] due to its causing potential of irritation and pain [23]. Therefore, the demand of new inhibitor of hyperpigmentation, particularly from marine algae, is rapidly increasing due to their safety as well psychological safety concern. Our results also evidence that arbutin adversely affected the physio-pathological phenomena of zebrafish embryos, but marine algal phlorotannins have proved to be safe as they have no adverse effect.

Inflammation is a fundamental innate immune response to perturbed tissue homeostasis. The prolonged exposure of arbutin showed pericadial edema with the elevation of inflammatory cytokines expression, however, E. cava phlorotannins did not show any alteration. Additionally, E. cava extract has shown in a previously study that it possesses excellent antioxidant activities and high phenolic content [9], thus making them potential candidates for dermatological application including melanin synthesis inhibition.

\section{Conclusion}

In summary, phlorotannins including PG, DK, and EK from E. cava were evaluated in regard to their potential efficacy as melanin formation inhibitors in zebrafish embryos. It can be surmised that the phlorotannins of marine alga is likely to be useful for the cosmetic and derma-medicinal industries.

\section{Acknowledgments}

This work was supported by grants from Basic Science Research Program through the National Research Foundation of Korea (NRF) funded by the Ministry of Science, ICT \& Future Planning (NRF2017R1D1A1B03033794). We thank Dr. Ann Kyle for editorial assistance.

\section{Conflict of interest}

The authors declare that there are no conflicts of interest.

\section{References}

1. Davis EC, Callender VD (2010) Postinflammatory hyperpigmentation: A review of the epidemiology, clinical features, and treatment options in skin of color. J Clin Aesthet Dermatol 3: 20-31.

2. Fujimoto N, Watanabe H, Nakatani T, Roy G, Ito A (1998) Induction of thyroid tumours in $(\mathrm{C} 57 \mathrm{BL} / 6 \mathrm{~N} \times \mathrm{C} 3 \mathrm{H} / \mathrm{N}) \mathrm{F} 1$ mice by oral administration of kojic acid. Food Chem Toxicol 36: 697-703.

3. Lee YS, Chang ZQ, Oh BC, Park SC, Shin SR, et al. (2007) Antioxidant activity, antiinflammatory activity, and whitening effects of extracts of Elaeagnus multiflora Thunb. J Med Food 10: 126-133.

4. Chang NF, Chen YS, Lin YJ, Tai TH, Chen AN, et al. (2017) Study of hydroquinone mediated cytotoxicity and hypopigmentation effects from UVB-irradiated arbutin and deoxyarbutin. Int J Mol Sci 18: 969.

5. Westerhof W, Kooyers TJ (2005) Hydroquinone and its analogues in dermatology - a potential health risk. J Cosmet Dermatol 4: 55-9.

6. Barbarino E, Lourenço SO (2005) An evaluation of methods for extraction and quantification of protein from marine macro- and microalgae. J Appl Phycol 17: 447-460.

7. Kuda T, Yano T, Matsuda N, Nishizawa M (2005) Inhibitory effects of laminaran and low molecular alginate against the putrefactive compounds produced by intestinal microflora in vitro and in rats. Food Chem 91: 745-749.

8. Heo SJ, Jeon YJ (2008) Radical scavenging capacity and cytoprotective effect of enzymatic digests of Ishige okamurae. J Appl Phycol 20: 1087-1095.

9. Heo SJ, Park PJ, Park EJ, Kim SK, Jeon YJ (2005) Antioxidant activity of enzymatic extracts from a brown seaweed Ecklonia cava by electron spin resonance spectrometry and comet assay. Eur Food Res Technol 221: 41-47.

10. Kuda T, Tsunekawa M, Goto H, Araki Y (2005) Antioxidant properties of four edible algae harvested in the Noto Peninsula, Japan. J Food Compost Anal 18: 625-633.

11. Wijesinghe WA, Ko SC, Jeon YJ (2011) Effect of phlorotannins isolated from Ecklonia cava on angiotensin I-converting enzyme (ACE) inhibitory activity. Nutr Res Pract 5: 93-100.

12. Kang SM, Cha SH, Ko JY, Kang MC, Kim D, et al. (2012) Neuroprotective effects of phlorotannins isolated from a brown alga, Ecklonia cava, against $\mathrm{H}_{2} \mathrm{O}_{2}$-induced oxidative stress in murine hippocampal HT22 cells. Environ Toxicol Pharmacol 34: 96-105.

13. Jung HA, Jin SE, Ahn BR, Lee CM, Choi JS (2013) Anti-inflammatory activity of edible brown alga Eisenia bicyclis and its constituents fucosterol and phlorotannins in LPS-stimulated RAW264.7 macrophages. Food Chem Toxicol 59: 199-206.

14. Kang MC, Cha SH, Wijesinghe WA, Kang SM, Lee SH, et al. (2013) Protective effect of marine algae phlorotannins against AAPH-induced oxidative stress in zebrafish embryo. Food chem 138: 950-955.

15. Heo SJ, Cha SH, Kim KN, Lee SH, Ahn G, et al. (2012) Neuroprotective effect of phlorotannin isolated from Ishige okamurae against $\mathrm{H}_{2} \mathrm{O}_{2}$-induced oxidative stress in murine hippocampal neuronal cells, HT22. Appl Biochem Biotechnol 166: 1520-1532.

16. Choi TY, Kim JH, Ko DH, Kim CH, Hwang JS, et al. (2007) Zebrafish as a new model for phenotype-based screening of melanogenic regulatory compounds. Pigment Cell Res 20: 120-127.

17. Cha SH, Lee JH, Kim EA, Shin CH, Jun HS, et al. (2017) Phloroglucinol accelerates the regeneration of liver damaged by $\mathrm{H}_{2} \mathrm{O}_{2}$ or MNZ treatment in zebrafish. RSC Advances 7: 46164-46170.

18. Ahn GN, Kim KN, Cha SH, Song CB, Lee J, et al. (2007) Antioxidant activities of phlorotannins purified from Ecklonia cava on free radical scavenging using ESR and $\mathrm{H}_{2} \mathrm{O}_{2}$-mediated DNA damage. Eur Food Res Technol 226: 71-79.

19. Kim E-A, Lee S-H, Lee J-H, Kang N, Oh J-Y, et al. (2016) A marine algal polyphenol, dieckol, attenuates blood glucose level by Akt pathway in alloxan induced hyperglycemia zebrafish model. RSC Advances 6: 83563 .

20. Cha SH, Ko SC, Kim D, Jeon YJ (2011) Screening of marine algae for potentia tyrosinase inhibitor: Those inhibitors reduced tyrosinase activity and melanin synthesis in zebrafish. $J$ Dermatol 38: 343-352.

21. Desmedt B, Courselle P, De Beer JO, Rogiers V, Grosber M, et al. (2016) Overview of skin whitening agents with an insight into the illegal cosmetic market in Europe. $J$ Eur Acad Dermatol Venereol 30: 943-950.

22. O'Donoghue JL (2006) Hydroquinone and its analogues in dermatology - a risk-benefit viewpoint. J Cosmet Dermatol 5: 196-203. 
Seon-Heui C (2020) Prolonged exposure of marine algal phlorotannins with whitening effect did not cause inflammatory hyperpigmentation in zebrafish larva

23. Tai Y, Wang C, Wang Z, Liang Y, Du J, et al. (2017) Involvement of transient receptor potential cation channel member A1 activation in the irritation and pain response elicited by skin-lightening reagent hydroquinone. Sci Rep 7: 7532.

24. Wang YH, Wen CC, Yang ZS, Cheng CC, Tsai JN, et al. (2009) Development of a whole-organism model to screen new compounds for sun protection. Mar Biotechnol 11: 419-429.

25. Hanamura T, Uchida E, Aoki H (2008) Skin-lightening effect of a polyphenol extract from Acerola (Malpighia emarginata DC.) fruit on UV-induced pigmentation. Biosci Biotechnol Biochem 72: 3211-3218.

26. Zhu W, Gao J (2008) The use of botanical extracts as topical skin-lightening agents for the improvement of skin pigmentation disorders. J Invest Derm Symp P 13: 20-24.

27. Shahidi F (2009) Nutraceuticals and functional foods: whole versus processed foods. Trends Food Sci Technol 20: 376-387.
28. Ibañez E, Herrero M, Mendiola JA, Castro-Puyana M(2012) Extraction and characterization of bioactive compounds with health benefits from marine resources: macro and micro algae, cyanobacteria, and invertebrates. Marine bioactive compounds 2012: 55-98.

29. Khotimchenko Y (2016) Tyrosinase inhibitors from marine algae. Br J Dermatol 175: 457-458.

30. Yoon NY, Eom TK, Kim MM, Kim SK (2009) Inhibitory effect of phlorotannins isolated from Ecklonia cava on mushroom tyrosinase activity and melanin formation in mouse B16F10 melanoma cells. J Agric Food Chem 57: 4124-4129.

31. Heo SJ, Ko SC, Cha SH, Kang DH, Park HS, et al. (2009) Effect of phlorotannins isolated from Ecklonia cava on melanogenesis and their protective effect against photooxidative stress induced by UV-B radiation. Toxicol in Vitro 23: 1123-1130.

32. Petit L, Piérard GE (2003) Analytic quantification of solar lentigines lightening by a $2 \%$ hydroquinone-cyclodextrin formulation. J Eur Acad Dermatol Venereol 17: 546-549.

Copyright: $\odot 2020$ Seon-Heui C. This is an open-access article distributed under the terms of the Creative Commons Attribution License, which permits unrestricted use, distribution, and reproduction in any medium, provided the original author and source are credited. 